\title{
Random super-prism wavelength meter
}

\author{
Michael Mazilu,* Tom Vettenburg, Andrea Di Falco, and Kishan Dholakia \\ SUPA, School of Physics and Astronomy, University of St. Andrews, North Haugh, St. Andrews KY16 9SS, UK \\ *Corresponding author: michael.mazilu@st-andrews.ac.uk
}

Received August 26, 2013; revised October 30, 2013; accepted November 11, 2013; posted November 22, 2013 (Doc. ID 196355); published December 20, 2013

\begin{abstract}
The speckle pattern arising from a thin random, disordered scatterer may be used to detect the transversal mode of an incident beam. On the other hand, speckle patterns originating from meter-long multimode fibers can be used to detect different wavelengths. Combining these approaches, we develop a method that uses a thin random scattering medium to measure the wavelength of a near-infrared laser beam with picometer resolution. The method is based on the application of principal component analysis, which is used for pattern recognition and is applied here to the case of speckle pattern categorization. @ 2013 Optical Society of America

OCIS codes: (030.6140) Speckle; (120.6160) Speckle interferometry; (070.4790) Spectrum analysis; (070.5010) Pattern recognition.

http://dx.doi.org/10.1364/OL.39.000096
\end{abstract}

Light propagation through time-dependent disordered or random media is generally regarded as a randomization process of the optical field destroying all the information contained within the initial beam. However, a coherent beam propagating in a stationary random medium yields a deterministic speckle pattern, while maintaining its initial spatial and temporal coherence. Such behavior has already been exploited in the design of several novel optical devices, e.g., to create focal spots using computergenerated holograms [1,2], to trap micro-particles [3] , and to coherently address plasmonic nanostructures [프. Key to these technological achievements is that the information content of the optical field is maintained when transmitted through a random medium. Thus the stationary wavefront randomization process can be used to robustly detect the state of the light field before scattering.

One way to understand this is to consider a beam propagating through a random diffuser. Before the random diffuser, a coherent beam can be seen as a superposition of many beamlets. After its propagation through the random diffuser, we observe an interference pattern between the constituent beamlets. This is a consequence of each beamlet having changed direction, spot size, and its phase relative to the other beamlets.

For the purposes of our present study, we can see this wavefront mixing process acting as a generalized interferometer, delivering a different speckle pattern for each different incident beam. This property can be used, for example, to simultaneously measure the azimuthal and radial modes of Laguerre-Gaussian beams $[\underline{5}, 6]$. It is interesting to consider the possibility of using this very same approach in random media to measure other key properties of the light field such as polarization state or wavelength. The use of wavemeters is ubiquitous in photonics and is conventionally realized using a 1D spatial mapping of the spectrum. Miniaturization of such devices would be highly advantageous, especially with regard to developing portable apparatus or even on-chip detection. To this end, recent work has shown that multimode fiber may be used to create wavefront randomization $[\underline{7}, \underline{8}]$ to act as a spectrometer. However, to measure the wavelength of a laser line with $8 \mathrm{pm}$ resolution, light has to be propagated along $20 \mathrm{~m}$ of multimode fiber, without any mechanical perturbation. Notably, it also has been separately recognized that spectral polarimetric measurements may be performed using the transmission matrix of random media [9]. Applications based upon the "lab-on-achip" principle require small integrated wavelength detectors. One way to achieve this is by propagating light through periodic structures such as a super-prism $[10,11]$ made from specially engineered photonic crystals. The optical dispersion of these crystals can deliver resolution of $0.4 \mathrm{~nm}$ at a wavelength of $1.5 \mu \mathrm{m}$ [12]. Random photonics devices also can be used to this effect [13], delivering only $0.75 \mathrm{~nm}$ resolution.

In this Letter, we show that, using the scattering properties of a remarkably simple thin diffuser, it is possible to detect the wavelength of a monochromatic beam to picometer precision, delivering an equivalent precision to a $20 \mathrm{~m}$ long multimode fiber. This can be understood by considering the decreased mean free path in such highly scattering media. This approach may be extended to even higher resolution through the use of an optical cavity placed around the randomizing medium. It also raises the prospect of ultra-compact spectrometers.

For the experiment, we used two laser sources: a tunable narrow linewidth Littman cavity diode laser system (Sacher Lasertechnik, $785 \mathrm{~nm}$, line width $<1 \mathrm{MHz}$, TEC510-0780-100) and a Ti:sapphire laser (Spectra-Physics, line width $0.5 \mathrm{GHz}$, tunable from $\lambda \approx 700$ to $1000 \mathrm{~nm}$, model 3900S). A HighFinesse/Ångstrom WS7 super-precision wavelength meter was used to perform independent calibration of each tunable laser source. The diode laser source was used in a narrow wavelength range study $(\delta \lambda \approx 0.5 \mathrm{~nm})$, whereas the Ti:sapphire laser source gave us the freedom to accurately tune and position the wavelength over a larger range. Both laser beams were filtered by coupling their outputs into single-mode fibers to avoid variability of the beam input beam shape when tuning the laser. To detect the speckle pattern, we used a CCD camera (Pike, Allied Vision Technologies, pixel pitch: $7.4 \mu \mathrm{m} \times 7.4 \mu \mathrm{m}$ ). Figure $1(\mathrm{c})$ shows a diagram of the experimental setup used for the data presented.

In our experiments, we considered two different geometries for the illumination of the random scatterer. In the first approach, we used a thin layer of random alumina particles under direct illumination. A small drop $(\approx 5 \mu \mathrm{l})$ of a commercially available solution (Agar Scientific) of 

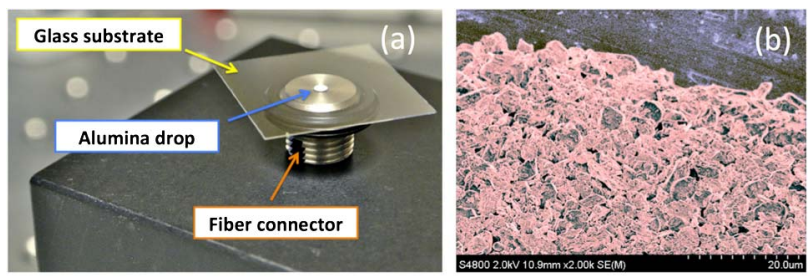

(c)

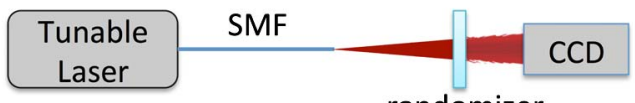

randomizer

Fig. 1. Experimental setup. (a) Photograph of a typical sample. (b) SEM image of the used alumina particles, with average size $5 \mu \mathrm{m}$. (c) Schematic of the experimental apparatus of the proposed spectrometer: $\mathrm{SMF}=$ single-mode fiber (Thorlabs P3-780A-FC-1) and CCD = charge-coupled camera.

alumina particles with a mean size of $5 \mu \mathrm{m}$ and deionized water was drop casted on a glass substrate. The glass slide was $160 \mu \mathrm{m}$ thick and had been previously cleaned with 5 min long immersions in Acetone, then Isopropanol in an ultrasonic bath, followed by oxygen-based plasma ashing at $100 \mathrm{~W}$. Care was taken to allow the deionized water to evaporate slowly in order to minimize the curling of the surface of the drying drop [see Figs. 1(a) and 1(b)]. The dried drop was measured to be $40 \mu \mathrm{m} \pm$ $10 \mu \mathrm{m}$ thick. A second approach, comprised of two highreflective laser-cavity mirrors within which a random diffuser was inserted, creating a randomized Fabry-Perot cavity.

At the heart of the random super-prism wave meter is the algorithm used to determine the wavelength corresponding to a given speckle pattern. This algorithm is similar to the one that we used for the detection of the azimuthal and radial mode indices of higher-order Laguerre Gaussian beams [5]. The procedure consists of two parts.

First, we calibrate the random super-prism wavelength meter by recording the speckle pattern for each wavelength that we want to detect. More precisely, we measure a number $N$ of patterns, where each speckle pattern is defined by a $2 \mathrm{D}$ array corresponding to the intensities measured by the CCD camera. Altogether, this delivers a 3D array corresponding to the intensities measured by the camera $A_{i j k}$, where the subscripts $i$ and $j$ are the pixel coordinate on the camera and $k$ an index distinguishing between different measurements. These different measurements either correspond to different wavelengths $\lambda$ or to multiple exposures having the same wavelength but in each case probing the fluctuations of the optical system. Figure 2(b) shows an example speckle pattern used in the calibration part of the experiment.

We then detect the largest variations between the different speckle patterns measured using the multivariate principal component analysis (PCA). In a first step, we subtract the average speckle image from every measured image $\hat{A}_{i j k}=A_{i j k}-\langle A\rangle_{i j k}$, where $\langle\cdot\rangle$ stands for the average over the index $k$. Further, we need to flatten the pixel coordinates part of the intensity array. This flattening process transforms the higher-order array into a $2 \mathrm{D}$ array $a_{m k}=\hat{A}_{i j k}$, where the integer index $1 \leq m \leq N$ corresponds to a unique mapping from the $(i, j)$ pair to the
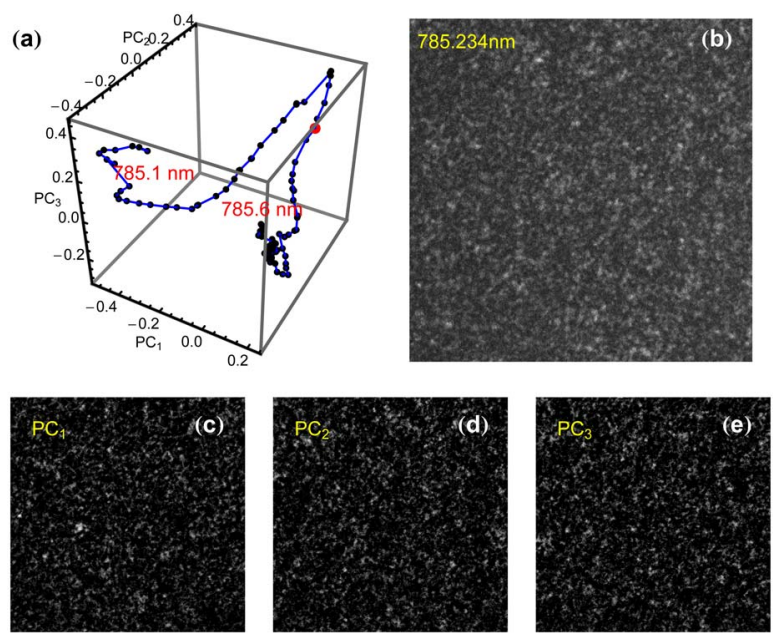

Fig. 2. Experimental measured wavelength using the alumina random super-prism in direct illumination (Media 1). (a) PCA decomposition of the detected speckle pattern as a function of the laser wavelength varying between 785.1 and $785.6 \mathrm{~nm}$. (b) An example of the far-field speckle pattern observed at $785.234 \mathrm{~nm}$. (c)-(e) First three principal components used in the decomposition.

linear index $m$. The principal components (PCs) are obtained by calculating the eigenvectors of the covariance matrix

$$
\mathbf{M}=\mathbf{a a}^{T},
$$

where the $T$ superscript stands for the matrix transposition. The covariance matrix $M$ is $N$ by $N$ sized. Each eigenvector has $N$ elements and can be recast in the $2 \mathrm{D}$ image form by exchanging the linear index $m$ to the pair index $(i, j)$. The eigenvector with the largest eigenvalue is called the first PC, the second largest to the second $\mathrm{PC}$ and so on. In general, only the first few PC are useful as they account for most variability in the system [14]. Additionally, the distribution of eigenvalues allows us to determine the number of degrees of freedom that the speckle pattern can access as the wavelength is varied. One method to calculate this number is by determining the number of eigenvalues above a certain threshold, which is determined by the signal-to-noise ratio or minimal measurable intensity. The larger the number of degrees of freedom, the greater the speckle pattern variability, and the better the wavelength resolution of the spectrometer.

The determination of the PC allows the representation of the speckle patterns in the PC space. Each measured speckle pattern can be decomposed into a static background (the average speckle pattern) and the weighted sum of a few PCs accounting for most variations. Figures 2(c), 2(d), and 2(e) show the first three PCs$P C_{1}, P C_{2}$, and $P C_{3}$ - which correspond to the first three degrees of freedom detectable by the speckle pattern. The three patterns look similar to each other; however, due to their eigenvector origin, these patterns are orthogonal to each other, and each one corresponds to an independent degree of freedom. Indeed, by construction, the matrix $M$ is Hermitian with non-negative eigenvalues whose eigenvectors are orthogonal to each other. 
After the decomposition, each speckle pattern can be represented by a small number of coefficients corresponding to the projection coefficients of the pattern onto the relevant PCs (in this case, the first eight PC). These coefficients are the coordinates of a point in the eight-dimensional PC space. Figure 2(a) (Media 1) shows the parametric curve described in the PC space (subspace defined by the first three PC) by the speckle pattern as the wavelength is varied over a range of $0.5 \mathrm{~nm}$. We observe in this figure that the parametric curve does not have a uniform length variation as a function of wavelength. This effect, in principle, adversely affects the uniformity of the wavelength resolution of our approach. However, in Fig. 2(a), we represent only the first three PC of the decomposition, and there are further five decomposition coefficients for each speckle pattern. Taking into account all eight PC decomposition coefficients greatly diminishes this effect and explains how the PCA method can deliver such a high resolution.

In the second part, we record the speckle pattern of an unknown wavelength. This pattern is decomposed in the previously calibrated PC space, and the wavelength can be established using, for example, the nearest neighbor Mahalanobis distance or linear regression classification methods. All these classification methods deliver perfect results (no error) if the detected wavelength is part of the calibration set. The red curve in Fig. $\underline{3}$ shows this in the case of the nearest-neighbor classification. This perfect result can be understood by considering our approach in the context of wavelength classification method. Indeed, in the case of speckle pattern fluctuations smaller than the step size used for the speckle pattern training set, the classification approach will deliver the nominal classification wavelength with no error at all. This also indicates that the resolution can be further improved by increasing the number of training wavelengths. On the other hand, the disadvantage of the classification approach is its inability to classify wavelengths that are not part of the original training set.

However, provided the parametric curve in the PC space is smooth, continuous, and locally linear, it also is possible to measure an unknown wavelength using, for

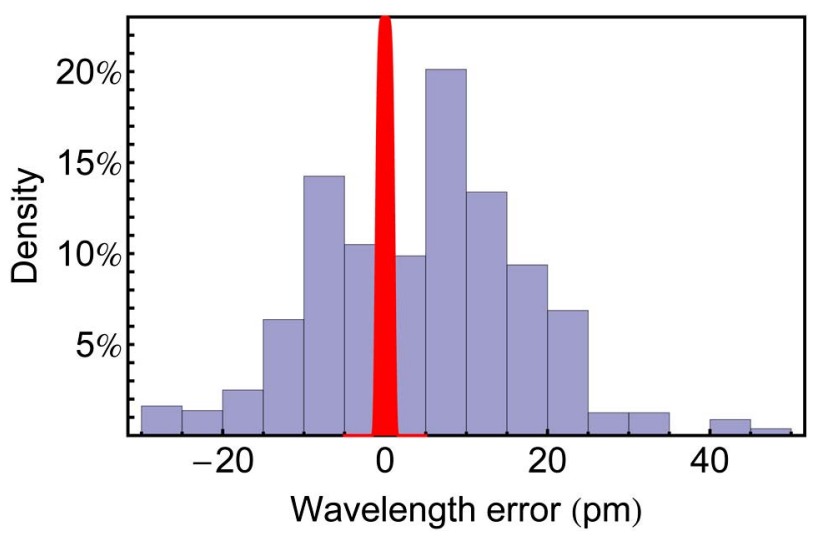

Fig. 3. Measured wavelength-error distribution in the case of the alumina drop in direct illumination. The bar chart shows the error distribution (bar chart) for the partial least-squares regression and (red curve) for the nearest-neighbor classification. The regression has a standard error deviation of $13 \mathrm{pm}$, and the nearest neighbor classification was achieved without error. example, partial least squares (PLS) regression in the PC space. We used this approach previously to characterize sub-wavelength displacements for optically based microscope sample stage stabilization [15]. Here we use PLS to detect the wavelength and determine its standard error deviation when the unknown wavelength is not necessarily part of the calibration set. Figure $\underline{3}$ shows that the standard deviation of the error is approximately $13 \mathrm{pm}$. This can be improved by considering smaller wavelength steps in the training set, yielding locally smaller deviations from the linear variation between each training step.

Further, there are routes to improve on the sensitivity of the random super-prism by including an optical feedback mechanism. This can be achieved by embedding the random scattering medium within a Fabry-Perot cavity. We have experimentally studied this possibility and have observed similar results as in the case of the thin layer of alumina particles. The main difference between the two devices was the much lower transmission intensity through the Fabry-Perot based device and the resulting need for an increased exposure time of the CCD detector. Otherwise, we did not experimentally observe any resolution improvement when using our specific Fabry-Perot cavity, which we attribute to insufficient reflectivity of the cavity mirrors. Figure 4 shows the numerical simulation (using Comsol) of this effect as a function of increasing the reflectivity of the cavity mirrors. This figure also shows a potential advantage of this configuration, i.e., the variability of the speckle pattern versus wavelength change is increased as the cavity provides more feedback. This improvement is ultimately limited by the loss in transmission efficiency. Further theoretical and experimental studies are necessary to establish the details of the link between device efficiency and wavelength resolution.

We also calibrated the random super-prism wave meter for use over a larger bandwidth using the Ti:sapphire laser system. The results are similar to the case of the

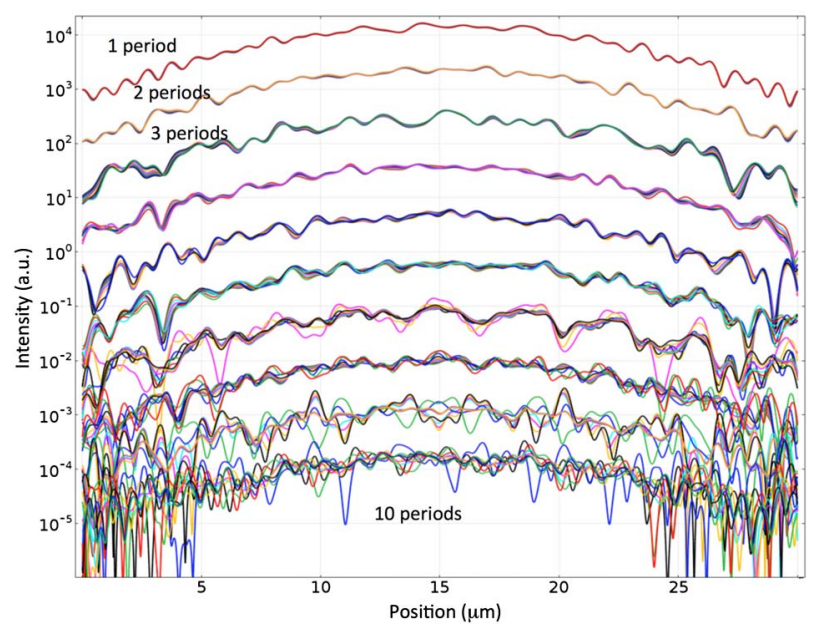

Fig. 4. Modeled speckle pattern variability considering a random diffuser placed inside a Fabry-Perot cavity composed of two distributed Brag reflectors having an increasing number of periods, i.e., increasing reflectivity. The different colors correspond to 10 different incident wavelengths chosen in a narrow wavelength range $(0.1 \mathrm{~nm})$. 
narrow bandwidth. In this case, it was possible to accurately detect the wavelength of the tunable laser varying from 795 to $805 \mathrm{~nm}$ with a resolution of $0.1 \mathrm{~nm}$. Finally, we remark that the approach presented here is generic from the perspective that it can be used to detect not only the wavelength, but, using suitable training, it can be used to detect the polarization state and shape of the incident beam. It is thus important to restrict the system so that the PCs are assigned to the optical variable of interest during training. Here we have used the single-mode fiber (SMF) to limit the system to a single variable, the wavelength. In effect, the SMF acts as the input slit in a monochromator, ensuring that at the output of the monochromator only variations in wavelength generate an intensity variation. However, replacing the SMF by a multimode fiber or pinhole would add to the wavelength variability of the speckle pattern the variations due to the beam shape $[5,6]$. An interesting issue arises when multiple parameters vary at the same time. Indeed, it is possible to generalize the training method to go beyond the detection of a single parameter such that multiple parameters can be measured at the same time. This includes not only beam-shape parameters but also laser polarization and multiple wavelengths simultaneously. The latter case leads to the construction of compact purpose-built spectrometers. Moreover, the simultaneous detection of changes in multiple beam parameters can give insight into a number of optical phenomena that all have an effect on the transmission of optical beams. Minute changes in these parameters can in effect be amplified by multiple scattering in the random diffuser and detected with high sensitivity.

We have demonstrated the use of a simple random medium as a wavelength meter with picometer resolution, exploiting the large number of degrees of freedom associated with the light transmission through this disordered medium. More precisely, we achieved $13 \mathrm{pm}$ resolution and a bandwidth of $10 \mathrm{~nm}$ at a wavelength of $800 \mathrm{~nm}$. The concept has been extended to random media placed within a cavity, thereby aiming to enhance the wavelength sensitivity at the expense of transmitted intensity.

We thank the UK Engineering and Physical Sciences Research Council for funding (EP/J004200/1). ADF is a EPSRC fellow (EP/I004602/1). Kishan Dholakia is a Royal Society-Wolfson Merit Award Holder.

\section{References}

1. I. Vellekoop and A. Mosk, Opt. Lett. 32, 2309 (2007).

2. I. M. Vellekoop, A. Lagendijk, and A. P. Mosk, Nat. Photonics 4, 320 (2010).

3. T. Čižmár, M. Mazilu, and K. Dholakia, Nat. Photonics 4, 388 (2010).

4. S. Kosmeier, A. C. De Luca, S. Zolotovskaya, A. Di Falco, and M. Mazilu, Sci. Rep. 3, 1808 (2013).

5. M. Mazilu, A. Mourka, T. Vettenburg, E. M. Wright, and K. Dholakia, Appl. Phys. Lett. 100, 231115 (2012).

6. A. Mourka, M. Mazilu, E. M. Wright, and K. Dholakia, Sci. Rep. 3, 1422 (2013).

7. B. Redding, S. M. Popoff, and H. Cao, Opt. Express 21, 6584 (2013).

8. B. Redding and H. Cao, Opt. Lett. 37, 3384 (2012).

9. T. W. Kohlgraf-Owens and A. Dogariu, Opt. Lett. 35, 2236 (2010)

10. L. Wu, M. Mazilu, T. Karle, and T. F. Krauss, Opt. Quantum Electron. 38, 915 (2002).

11. A. Di Falco, C. Conti, and G. Assanto, J. Lightwave Technol. 22, 1748 (2004).

12. T. Baba and T. Matsumoto, Appl. Phys. Lett. 81, 2325 (2002).

13. B. Redding, S. F. Liew, R. Sarma, and H. Cao, Nat. Photonics 7, 746 (2013).

14. P. Francis and B. Wills, Quas. Cosmol. 162, 363 (1999).

15. X. Tsampoula, M. Mazilu, T. Vettenburg, F. Gunn-Moore, and K. Dholakia, Photon. Res. 1, 42 (2013). 